\title{
THE EXPONENTIAL DECAY RATE OF THE LOWER BOUND FOR THE FIRST EIGENVALUE OF COMPACT MANIFOLDS
}

\author{
MORRIS KALKA, ELIZABETH MANN, DAGANG YANG, AND ALEKSEY ZINGER
}

\begin{abstract}
This paper provides the optimal exponential decay rate of the lower bound for the first positive eigenvalue of the Laplacian operator on a compact Riemannian manifold with a negative lower bound on the Ricci curvature and with large diameter. For manifolds with boundary, suitable convexity conditions are assumed.
\end{abstract}

\section{INTRODUCTION}

The aim of this paper is to provide the asymptotically sharp exponential decay rate for the lower bound of the first positive eigenvalue of the Laplacian operator on compact Riemannian manifolds.

Estimates of the first eigenvalue are of particular interest in geometric analysis. For example, a sharp lower bound on the first eigenvalue gives a sharp upper bound for the constant in the Poincaré inequality.

For manifolds with positive Ricci curvature, $\operatorname{Ric}_{M} \geq(n-1) k>0$, the first result, $\lambda_{1} \geq n k$, was obtained by Lichnerowicz [LZ]. This bound is sharp; it is achieved if and only if the manifold is a round sphere, as shown by Obata in [O]. In terms of diameter, the third author recently proved in $[\mathrm{Y}]$ that $\lambda_{1} \geq(n-1) k / 4+\pi^{2} / d^{2}$.

For manifolds with non-negative Ricci curvature, Li and Yau obtained the lower bound $\lambda_{1} \geq \frac{\pi^{2}}{2 d^{2}}$, where $d$ is the diameter of the manifold, using the gradient estimate technique they developed in $[\mathrm{L}, \mathrm{LY}]$. Zhong and Yang $[\mathrm{ZY}]$ improved this bound to $\frac{\pi^{2}}{d^{2}}$, which is known to be sharp.

When the Ricci curvature is bounded below by $-(n-1) k$ for $k \geq 0, \mathrm{Li}$ and Yau in $[\mathrm{L}, \mathrm{LY}]$ obtained the bound:

$$
\lambda_{1} \geq \frac{\exp \left(-\left\{1+\sqrt{1+4(n-1)^{2} k d^{2}}\right\}\right)}{(n-1) d^{2}} .
$$

These results display the interesting general principle that the scaling invariant $d^{2} \lambda_{1}$ can be estimated from below in terms of the dimension $n$ and the scaling invariant $d^{2} k$ of the manifold. For manifolds with large diameter $d$, the Li-Yau estimate shows that $d^{2} \lambda_{1}$ has a lower bound which decays exponentially in $d$ with decay rate $2(n-1) \sqrt{k}$. More background information on first eigenvalue estimates can be found in $[\mathrm{SY}]$.

1991 Mathematics Subject Classification. 53C and 58G.

Key words and phrases. First eigenvalue, eigenfunction, gradient estimate, Ricci curvature, geometric analysis, Riemannian manifold.

Research partially supported by NSF grant number DMS-9531627. The research was done at the REU program at Tulane University. 
In this paper, we show that the optimal decay rate is given by $\frac{1}{2}(n-1) \sqrt{k}$. The main results of this article are summarized in the following theorem.

Theorem Let $(M, g)$ be a compact connected Riemannian $n$-manifold with diameter $d$ and Ricci curvature bounded below as $\operatorname{Ric}_{M} \geq-(n-1) k$, for some constant $k \geq 0$.

1) If $\partial M$ is empty, and $\lambda_{1}$ is the first eigenvalue of the Laplacian, then

$$
\lambda_{1} \geq \frac{\sqrt{k}}{10 d} \exp \left(-\frac{1}{2}(n-1) \sqrt{k} d\right) .
$$

2) If $\partial M$ is nonempty and weakly convex, and $\lambda_{N, 1}$ is the first eigenvalue of the Laplacian under the Neumann condition, then

$$
\lambda_{1} \geq \frac{\sqrt{k}}{10 d} \exp \left(-\frac{1}{2}(n-1) \sqrt{k} d\right) .
$$

3) If $\partial M$ is nonempty and of nonnegative mean curvature in the direction of the outer normal; $I$ is the inscribed radius, and $\lambda_{D, 1}$ is the first eigenvalue of the Laplacian under the Dirichlet condition, then

$$
\lambda_{1} \geq \frac{\sqrt{k}}{12 I} \exp (-(n-1) \sqrt{k} I) .
$$

More detailed bounds are given in Theorems 1, 2 and 3. We consider closed manifolds in Section 2 and manifolds with boundary in Section 3. In Section 4, we construct examples demonstrating that the exponential decay rate of the lower bound is sharp. However, it is not clear whether the lower order terms in our eigenvalue bound are also sharp.

Throughout this paper, an $n$-manifold will denote a smooth manifold of dimension $n \geq 2$. The first positive eigenvalue will be referred to as the first eigenvalue.

\section{Closed Manifolds}

Theorem 1 Let $(M, g)$ be a compact connected Riemannian $n$-manifold with $\partial M=\emptyset$, diameter $d$, and Ricci curvature bounded below as $\operatorname{Ric}_{M} \geq-(n-1) k$, for some constant $k \geq 0$. Then, the first eigenvalue $\lambda_{1}$ of the Laplacian satisfies

$$
\begin{aligned}
\lambda_{1} \geq & \frac{48}{(3 \pi+1)(n+1) d^{2}}\left\{1+\left[1+\frac{1}{4}(n-1)^{2} k d^{2}\right]^{1 / 2}\right\} \\
& \times \exp \left(-\left\{1+\left[1+\frac{1}{4}(n-1)^{2} k d^{2}\right]^{1 / 2}\right\}\right) .
\end{aligned}
$$

Proof. Let $v$ be the first eigenfunction such that

$$
\sup v=1, \quad \inf v=-b, \quad 0<b \leq 1 .
$$

Put

$$
u=2 \beta \cdot \frac{2 v-(1-b)}{1+b}
$$

where $0<\beta<\frac{\pi}{4}$. Then,

$$
\sup u=2 \beta, \quad \inf u=-2 \beta, \quad \Delta u=-\lambda_{1}(u+\epsilon), \quad \text { where } \epsilon=2 \beta \cdot \frac{1-b}{1+b} .
$$


Define a function $G: M \rightarrow \mathbb{R}$ by

$$
G(x)=\frac{|\nabla u(x)|^{2}}{\cos ^{2} u(x)} .
$$

Note that $G$ is an everywhere defined smooth function due to (1). We first obtain the following upper bound for $G$ :

$$
G \leq(n-1)^{2} k+[(n-1)+(n+1) \pi \tan 2 \beta] \lambda_{1} .
$$

Let $x_{0}$ be a point of $M$ at which $G$ achieves a maximum. Then,

$$
\nabla G\left(x_{0}\right)=0 \quad \text { and } \quad \Delta G\left(x_{0}\right) \leq 0 .
$$

Given a normal orthonormal frame $\left\{e_{i}\right\}$ for $T M$ in a neighborhood of a point of $M$ and a smooth function $f$ on $M$, denote $\left(\nabla^{k} f\right)_{i_{1} \ldots i_{k}}$ by $f_{i_{1} \ldots i_{k}}$, where $\nabla$ is the covariant differential operator of the Levi-Civita connection. For convenience, all functions mentioned below will denote their value at $x_{0}$, unless stated otherwise. Choose a normal orthonormal frame $\left\{e_{i}\right\}$ for $T M$ around $x_{0}$ such that $u_{i}=0$ for $i \neq 1$; then $u_{1} \neq 0$ (or else $G$ is identically 0 , and $u$ is constant). The first equation of (4) implies $G_{i}=0$, and therefore,

$$
\sum_{j} u_{j i} u_{j}=-|\nabla u|^{2} u_{i} \tan u \quad \forall i .
$$

In particular, $u_{11}=-|\nabla u|^{2} \tan u$. Taking the Laplacian of the function $G \cdot \cos ^{2} u$ and applying the product rule and Bochner's formula, (4) gives

$$
\begin{aligned}
0 \geq & (\Delta G) \cos ^{2} u=\Delta\left(|\nabla u(x)|^{2}\right)-2 \nabla G \cdot \nabla\left(\cos ^{2} u\right)-G \Delta\left(\cos ^{2} u\right) \\
= & 2\left\{\sum_{i, j} u_{i j}^{2}+\nabla u \cdot \nabla(\Delta u)+\operatorname{Ric}(\nabla u, \nabla u)\right\} \\
& -G\left\{-2|\nabla u|^{2} \cos 2 u-(\Delta u) \sin 2 u\right\} \\
\geq & 2\left\{\sum_{i, j} u_{i j}^{2}-\lambda_{1}|\nabla u|^{2}-(n-1) k|\nabla u|^{2}\right\} \\
& -G\left\{-2|\nabla u|^{2} \cos 2 u+\lambda_{1}(u+\epsilon) \sin 2 u\right\} .
\end{aligned}
$$

Using the Schwartz inequality,

$$
\begin{aligned}
\sum_{i, j} u_{i j}^{2} & \geq u_{11}^{2}+\sum_{i=2}^{n} u_{i i}^{2} \geq u_{11}^{2}+\frac{1}{n-1}\left(\sum_{i=2}^{n} u_{i i}\right)^{2} \\
& =u_{11}^{2}+\frac{1}{n-1}\left(\Delta u-u_{11}\right)^{2}=u_{11}^{2}+\frac{1}{n-1}\left(\lambda_{1}(u+\epsilon)+u_{11}\right)^{2} \\
& \geq \frac{n}{n-1} u_{11}^{2}+\frac{2}{n-1} u_{11} \lambda_{1}(u+\epsilon)
\end{aligned}
$$


Substituting into equation (6), we obtain

$$
\begin{aligned}
0 \geq & \left(-\lambda_{1}|\nabla u|^{2}-(n-1) k|\nabla u|^{2}\right) \\
& +\left(\frac{n}{n-1}|\nabla u|^{4} \tan ^{2} u-\frac{2}{n-1} \lambda_{1}|\nabla u|^{2}(u+\epsilon) \tan u\right) \\
& -G\left\{-|\nabla u|^{2} \cos 2 u+\frac{1}{2} \lambda_{1}(u+\epsilon) \sin 2 u\right\} \\
= & \left\{\frac{n}{n-1} \tan ^{2} u+\frac{\cos 2 u}{\cos ^{2} u}\right\}|\nabla u|^{4} \\
& -\left\{(n-1) k+\left[1+\left(1+\frac{2}{n-1}\right)(u+\epsilon) \tan u\right] \lambda_{1}\right\}|\nabla u|^{2} \\
= & \frac{1+(n-2) \cos ^{2} u}{n-1} \cdot \frac{|\nabla u|^{4}}{\cos ^{2} u} \\
& -\left\{(n-1) k+\left[1+\frac{n+1}{n-1}(u+\epsilon) \tan u\right] \lambda_{1}\right\}|\nabla u|^{2} .
\end{aligned}
$$

Dividing both sides of the above by $|\nabla u|^{2}$ yields

$$
\begin{aligned}
G & \leq \frac{(n-1) k+\left[1+\frac{n+1}{n-1}(u+\epsilon) \tan u\right] \lambda_{1}}{\left[1+(n-2) \cos ^{2} u\right] /(n-1)} \\
& \leq(n-1)^{2} k+[(n-1)+(n+1) \pi \tan 2 \beta] \lambda_{1},
\end{aligned}
$$

as desired.

Now choose points $x_{1}$ and $x_{2}$ of the compact manifold $M$ such that $u\left(x_{1}\right)=\inf u$ and $u\left(x_{2}\right)=\sup u$. Let $\gamma$ be a minimal geodesic from $x_{1}$ to $x_{2}$. Then,

$$
\begin{aligned}
\ln \tan ^{2}\left(\frac{\pi}{4}+\beta\right) & =\int_{-2 \beta}^{2 \beta} \frac{d u}{\cos u} \leq \int_{\gamma} \sqrt{G} d s \\
& \leq\left[(n-1)^{2} k+((n-1)+(n+1) \pi \tan 2 \beta) \lambda_{1}\right]^{1 / 2} d \\
& \leq\left[(n-1)^{2} k+(n+1)(1+\pi \tan 2 \beta) \lambda_{1}\right]^{1 / 2} d \\
& \left.=\left[(n-1)^{2} k+(n+1)(1 / \tan 2 \beta+\pi) \lambda_{1} \tan 2 \beta\right)\right]^{1 / 2} d \\
& \left.\leq\left[(n-1)^{2} k+(n+1) \frac{3 \pi+1}{3} \lambda_{1} \tan 2 \beta\right)\right]^{1 / 2} d .
\end{aligned}
$$

The last inequality above is obtained by assuming that $\tan 2 \beta \geq 3$. Solving for $\lambda_{1}$,

$$
\begin{aligned}
\lambda_{1} & \geq \frac{3}{(3 \pi+1)(n+1)} \cot 2 \beta\left\{\frac{4}{d^{2}} \ln ^{2} \tan \left(\frac{\pi}{4}+\beta\right)-(n-1)^{2} k\right\} \\
& \geq \frac{6}{(3 \pi+1)(n+1)} \tan \left(\frac{\pi}{4}-\beta\right)\left\{\frac{4}{d^{2}} \ln ^{2} \tan \left(\frac{\pi}{4}-\beta\right)-(n-1)^{2} k\right\} .
\end{aligned}
$$

The right-hand side of (11) is maximized when

$$
\ln \tan \left(\frac{\pi}{4}-\beta\right)=-1-\sqrt{1+\frac{1}{4}(n-1)^{2} k d^{2}} .
$$

For this value of $\beta$, one obtains the desired lower bound. Observe that

$$
\tan 2 \beta=\frac{1-\tan ^{2}\left(\frac{\pi}{4}-\beta\right)}{2 \tan \left(\frac{\pi}{4}-\beta\right)}=\frac{1}{2}\left(\frac{1}{\tan \left(\frac{\pi}{4}-\beta\right)}-\tan \left(\frac{\pi}{4}-\beta\right)\right) \geq \frac{1}{2}\left(e^{2}-e^{-2}\right) \geq 3,
$$


so the last inequality in (10) is valid $\square$

Note that if one puts $u=2 \beta v$, where $v$ and $\beta$ are as before, then the same argument as in the above proof gives the following result, analogous to equation (9).

Corollary 1 Let $(M, g)$ be a closed compact connected Riemannian $n$-manifold with Ricci curvature bounded below as $\operatorname{Ric}_{M} \geq-(n-1) k$, for some constant $k \geq 0$. If $u$ is an eigenfunction of the Laplacian with eigenvalue $\lambda$ such that $\sup |u|=2 \beta$, $0<\beta<\frac{\pi}{4}$, then

$$
|\nabla u| \leq\left[(n-1)^{2} k+\left[(n-1)+(n+1) \frac{\pi}{2} \tan 2 \beta\right] \lambda\right]^{1 / 2} \cos u
$$

Remark The above gradient estimate applies to manifolds with boundary under either the Neumann or Dirichlet boundary conditions, as long as sufficient conditions are imposed on the boundary of $M$ to ensure that $\nabla G=0$ and $\Delta G \leq 0$ at any point of a maximum of $G$. Once these two facts have been established, the proof is the same as before.

\section{Manifolds With Boundary}

Definition 1 If $M$ is a manifold with boundary, the boundary $\partial M$ is said to be weakly convex if the second fundamental form $\Pi$ on $\partial M$ with respect to the outer normal is nonnegative definite.

Theorem 2 Let $(M, g)$ be a compact connected Riemannian $n$-manifold with weakly convex boundary, diameter $d$, and Ricci curvature bounded below as $\operatorname{Ric}_{M} \geq$ $-(n-1) k$, for some constant $k \geq 0$. Then, the first eigenvalue $\lambda_{N, 1}$ of the Laplacian with respect to the Neumann boundary condition satisfies

$$
\begin{aligned}
\lambda_{N, 1} \geq & \frac{48}{(3 \pi+1)(n+1) d^{2}}\left\{1+\left[1+\frac{1}{4}(n-1)^{2} k d^{2}\right]^{1 / 2}\right\} \\
& \times \exp \left(-\left\{1+\left[1+\frac{1}{4}(n-1)^{2} k d^{2}\right]^{1 / 2}\right\}\right) .
\end{aligned}
$$

Proof. Let $v, u, \beta, G$, and $x_{0}$ be as in the proof of Theorem 1 . If $x_{0}$ is in the interior of $M$, then we obtain the same gradient estimate (3) as in the proof of Theorem 1.

Suppose $x_{0} \in \partial M$. Choose an orthonormal frame $\left\{e_{i}\right\}$ for $T M$ around $x_{0}$ such that $e_{1}$ is the outward normal to the boundary of $M$. The Neumann boundary condition implies that $u_{1}=0$. At $x_{0}$ we must have $G_{1} \geq 0$ and $G_{2}=\ldots=G_{n}=0$. Note that

$$
u_{i 1}=e_{i}\left(e_{1} u\right)-\left(\nabla_{e_{i}} e_{1}\right) u=0-\sum_{j=1}^{n}\left\langle\nabla_{e_{i}} e_{1}, e_{j}\right\rangle e_{j} u=-\sum_{j=2}^{n} \Pi_{i j} u_{j}
$$


We now compute $G_{1}$ at $x_{0}$ :

$$
\begin{aligned}
0 \leq G_{1} & =\frac{2}{\cos ^{2} u}\left(\sum_{i} u_{i 1} u_{i}+|\nabla u|^{2} u_{1} \tan u\right) \\
& =\frac{2}{\cos ^{2} u}\left(\sum_{i} u_{i 1} u_{i}\right)=-\frac{2}{\cos ^{2} u} \sum_{i, j=1}^{n} \Pi_{i j} u_{i} u_{j} \leq 0,
\end{aligned}
$$

since the boundary is weakly convex. It follows that at $x_{0}, G_{1}=0$ and thus $G_{11} \leq 0$ (since $G$ reaches a maximum at $x_{0}$ ). Thus, at $x_{0}$

$$
\Delta G=G_{11}+m G_{1}+\bar{\Delta} G=G_{11}+\bar{\Delta} G \leq 0,
$$

where $m$ is the mean curvature of the boundary in the direction of the outer normal at $x_{0}$, and $\bar{\Delta}$ is the Laplacian on the boundary with respect to the induced metric. Since $\nabla G=0$ and $\Delta G \leq 0$ at every maximum of $G$, the proof of Theorem 1 shows that the gradient estimate (3) is still valid.

The rest of the proof of Theorem 1 can now be followed to obtain the same bound for the first eigenvalue

Definition 2 The inscribed radius I of a manifold with boundary is the greatest distance from any point to the boundary.

Theorem 3 Let $(M, g)$ be a compact Riemannian manifold with inscribed radius $I$, boundary of non-negative mean curvature $m$ in the direction of the outward normal, and Ricci curvature bounded below as $\operatorname{Ric}_{M} \geq-(n-1) k$, for some constant $k \geq 0$. Then the first eigenvalue with respect to the Dirichlet boundary condition satisfies:

$$
\begin{aligned}
\lambda_{D, 1} \geq & \frac{24}{(3 \pi+2)(n+1) I^{2}}\left\{1+\left[1+(n-1)^{2} k I^{2}\right]^{1 / 2}\right\} \\
& \times \exp \left(-\left\{1+\left[1+(n-1)^{2} k I^{2}\right]^{1 / 2}\right\}\right) .
\end{aligned}
$$

Proof. Let $v$ be the first eigenfunction satisfying the Dirichlet boundary condition, $\left.v\right|_{\partial M} \equiv 0, \operatorname{such}$ that $\sup v=1$. Put $u=2 \beta v$ for $0<\beta<\frac{\pi}{4}$. Define $G$ as in the proof of Theorem 1. Since $0 \leq u<\frac{\pi}{2}, G$ is again everywhere defined. We first show that the gradient estimate of Corollary 1 is still valid.

Let $x_{0}$ be a maximum of $G$. If $x_{0}$ is in the interior of $M$, then the validity of Corollary 1 is immediate from the Remark.

We now consider the possibility that $x_{0}$ is on the boundary of $M$. Choose an orthonormal frame $\left\{e_{i}\right\}$ for $T M$ around $x_{0}$ such that $e_{1}$ is the outward normal to the boundary of $M$. Since $u \equiv 0$ on the boundary, at $x_{0}$ we have $u_{2}=\ldots=u_{n}=0$ and

$$
0=-\lambda_{D, 1} u=\Delta u=u_{11}+m u_{1}+\bar{\Delta} u=u_{11}+m u_{1} .
$$

In addition, since $G$ achieves a maximum at $x_{0}, G_{1}\left(x_{0}\right) \geq 0$ and $G_{2}\left(x_{0}\right)=\ldots=$ $G_{n}\left(x_{0}\right)=0$. Evaluating $G_{1}$ at $x_{0}$, one obtains

$$
0 \leq G_{1}=\frac{1}{\cos ^{3} u}\left[\sum_{i=1}^{n} 2 u_{i} u_{i 1} \cos u+2|\nabla u|^{2} u_{1} \sin u\right]=2 u_{1} u_{11}=-2 m u_{1}^{2} \leq 0,
$$

since the mean curvature is nonnegative. Thus, $G_{1}\left(x_{0}\right)=0$ and $\Delta G\left(x_{0}\right) \leq 0$, as in the proof of Theorem 2. It follows by the Remark that the gradient estimate in Corollary 1 is still valid. 
Now choose a point $x_{1}$ at which $u\left(x_{1}\right)=2 \beta$, and let $\gamma$ be a minimizing geodesic from $x_{1}$ to the closest point on the boundary. The length of $\gamma$ is less than or equal to the inscribed radius $I$, and we obtain:

$$
\begin{aligned}
\ln \tan \left(\frac{\pi}{4}+\beta\right) & =\int_{0}^{2 \beta} \frac{d u}{\cos u} \leq \int_{\gamma} \sqrt{G} d s \\
& \leq\left[(n-1)^{2} k+\left[(n-1)+(n+1) \frac{\pi}{2} \tan 2 \beta\right] \lambda_{D, 1}\right]^{1 / 2} I \\
& \leq\left[(n-1)^{2} k+\left[(n+1) \frac{3 \pi+2}{6} \tan 2 \beta\right] \lambda_{D, 1}\right]^{1 / 2} I,
\end{aligned}
$$

under the assumption that $\tan 2 \beta \geq 3$ as in the proof of Theorem 1 . Solving for $\lambda_{D, 1}$ as before,

$$
\begin{aligned}
\lambda_{D, 1} & \geq \frac{6}{(3 \pi+2)(n+1)} \cot 2 \beta\left\{\frac{1}{I^{2}} \ln ^{2} \tan \left(\frac{\pi}{4}+\beta\right)-(n-1)^{2} k\right\} \\
& \geq \frac{12}{(3 \pi+2)(n+1)} \tan \left(\frac{\pi}{4}-\beta\right)\left\{\frac{1}{I^{2}} \ln ^{2} \tan \left(\frac{\pi}{4}-\beta\right)-(n-1)^{2} k\right\} .
\end{aligned}
$$

The desired result is obtained by maximizing the right-hand side of (13) with respect to $\tan \left(\frac{\pi}{4}-\beta\right)$ in the range $0<\tan \left(\frac{\pi}{4}-\beta\right) \leq e^{-2}$

\section{EXAmples}

In this section, we show that the exponential decay rates of the first eigenvalue bounds of Theorems 1, 2 and 3 are sharp. The manifolds described below have the shape of a hyperbolic dumbbell, akin to Calabi's dumbbell example as illustrated in Cheeger's work [C]. We first construct an example for the Neumann boundary problem.

Example 1 There exists a family of compact Riemannian $n$-manifolds with convex boundary $\left\{\left(M_{T}, g_{T}\right)\right\}, T \in(2, \infty)$, with diameter $d_{T} \rightarrow \infty$ as $T \rightarrow \infty$, and $\operatorname{Ric}_{M_{T}} \geq-(n-1)$ such that

$$
\lambda_{N, 1} \leq B_{n} \exp \left(-\frac{1}{2}(n-1) d_{T}\right) .
$$

Recall that the first eigenvalue $\lambda_{N, 1}$ is characterized as

$$
\lambda_{N, 1}=\inf \frac{\int_{M}|\nabla \phi|^{2}}{\int_{M} \phi^{2}}
$$

where $\int_{M} \phi=0, \phi \in L_{1}^{2}(M)$, and $\phi$ satisfies the Neumann boundary condition.

We begin with the infinite hyperbolic dumbbell, namely, the product manifold $\mathbb{R} \times \mathbb{S}^{\ltimes-\nVdash}$ with the metric $g=d t^{2}+\cosh ^{2}(t) \bar{g}$, where $\bar{g}$ denotes the standard metric on $S^{n-1}$. This manifold has constant sectional curvature -1 on all tangent planes containing $\partial_{t}$, and sectional curvature $>-1$ on all other tangent planes. The manifold $\left(M_{T}, g_{T}\right)$ is obtained by truncating this infinite hyperbolic dumbbell at $t= \pm T$.

To determine the diameter of this manifold, note that the greatest distance between any point and the submanifold $0 \times S^{n-1}$ is $T$, and the greatest distance between any two points on that submanifold is $\pi$. Thus, the diameter $d$ satisfies 
$2 T \leq d \leq 2 T+\pi$. As $T$ becomes large, $d / T$ approaches 2 . Notice that the boundary of $M_{T}$ is strictly convex since the derivative of $\cosh t$ at $T$ is positive.

For convenience, we henceforth refer to the manifold $\left(M_{T}, g_{T}\right)$ as $M$. Define the function $\phi: M \rightarrow \mathbb{R}$ as follows:

$$
\phi(t)= \begin{cases}-1 & t \in[-T,-1] \\ t & t \in[-1,1] \\ 1 & t \in[1, T] .\end{cases}
$$

Note that this function integrates to 0 on $M$, as desired.

For the function $\phi$, we have

$$
\begin{aligned}
\int_{M}|\nabla \phi|^{2} & =\int_{S^{n-1}} \int_{-1}^{1}\left(\phi^{\prime}(t)\right)^{2} \cosh ^{n-1}(t) d t d \omega \\
& =A_{n} \operatorname{vol}\left(S^{n-1}\right),
\end{aligned}
$$

where $A_{n}=\int_{-1}^{1} \cosh ^{n-1}(t) d t$. Note that

$$
\begin{aligned}
\int_{M} \phi^{2} & \geq 2 \operatorname{vol}\left(S^{n-1}\right) \int_{1}^{T} 1 \cdot \cosh ^{n-1}(t) d t \\
& \geq \frac{\operatorname{vol}\left(S^{n-1}\right)}{2^{n-1}(n-1)} e^{(n-1) T} \\
& \geq \frac{\operatorname{vol}\left(S^{n-1}\right)}{2^{n-1}(n-1)} e^{-(n-1) \pi / 2} e^{(n-1) d / 2} .
\end{aligned}
$$

This gives the upper bound

$$
\begin{aligned}
\lambda_{1} & \leq \frac{\int_{M}|\nabla \phi|^{2}}{\int_{M} \phi^{2}} \\
& \leq 2^{n-1} A_{n}(n-1) e^{(n-1) \pi / 2} e^{-(n-1) d / 2} \\
& =B_{n} e^{-(n-1) d / 2} .
\end{aligned}
$$

Thus, the exponential decay rate in the lower bound of Theorem 2 is sharp

Example 2 There exists a family of compact closed Riemannian $n$-manifolds $\left\{\left(M_{T}^{\prime}, g_{T}^{\prime}\right)\right\}, T \in(2, \infty)$, with diameter $d_{T} \rightarrow \infty$ as $T \rightarrow \infty$, and $\operatorname{Ric}_{M_{T}^{\prime}} \geq-(n-1)$ such that

$$
\lambda_{1} \leq B_{n} \exp \left(-\frac{1}{2}(n-1) d_{T}\right) .
$$

We start with the family $\left\{\left(M_{T}, g_{T}\right)\right\}$ of Example 1. $M_{T}^{\prime}$ is the double of $M_{T}$ constructed by identifying two copies of $M_{T}$ along their boundaries. The metric $g_{T}^{\prime}$ is obtained by modifying the metric $g_{T}$ on $[-T,-T+1)$ and $(T-1, T]$, so that $g_{T}^{\prime}=d t^{2}+f^{2}(t) \bar{g}$ with $f(-t)=f(t), f(t) \geq \cosh (T-1)$ for $t \in[T-1, T]$, $0 \leq f^{\prime}(t) \leq f(t)$ for $t \in[T-1, T], f^{\prime \prime}(t) \leq f(t)$, and $f^{(k)}(T)=0$ for all $k \geq 1$ (see Figure 1; note that the outside ends are identified, and that the metric is not induced by the embedding in $\mathbb{R}^{\ltimes+\nVdash}$ ). Under these conditions on $f$, the lower curvature bound remains unchanged. The upper bound for the diameter still holds, and both integrals are doubled to yield the same upper bound on $\lambda_{1} \square$ 


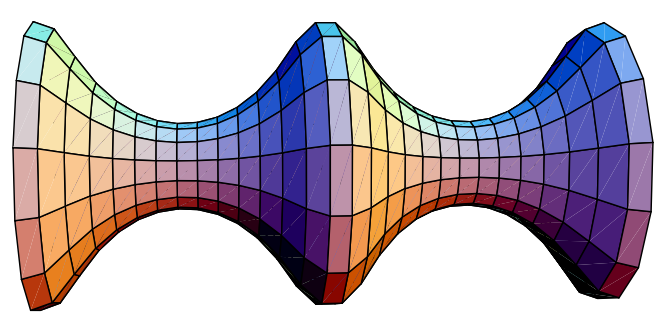

Figure 1. The doubled hyperbolic dumbbell.

Example 3 There exists a family of compact Riemannian $n$-manifolds $\left\{\left(M_{T}^{\prime \prime}, g_{T}^{\prime \prime}\right)\right\}$, with boundary of zero mean curvature, $T \in(2, \infty)$, with inscribed radius $I_{T} \rightarrow \infty$ as $T \rightarrow \infty$, and $\operatorname{Ric}_{M_{T}^{\prime}} \geq-(n-1)$ such that

$$
\lambda_{D, 1} \leq B_{n} \exp \left(-(n-1) I_{T}\right) .
$$

Let $M_{T}^{\prime \prime}=\left\{x \in M_{T}^{\prime} \mid \phi(x) \geq 0\right\}$, and let $g_{T}^{\prime \prime}$ be the restriction of $g_{T}^{\prime}$ to $M_{T}^{\prime \prime}$. Then, the boundary of $M_{T}^{\prime \prime}$ consists of the two necks of the double hyperbolic dumbbell. Because $f^{\prime}(0)=0$, the boundary is totally geodesic. Observe that the inscribed radius is exactly $\mathrm{T}$, and the calculations of Example 1 still hold

Note that our estimate has the form

$$
\lambda_{1} \geq \frac{1}{10 d} \exp \left(-\frac{1}{2}(n-1) d\right)
$$

for $k=1$, while for the examples in this section,

$$
\lambda_{1} \leq B_{n} \exp \left(-\frac{1}{2}(n-1) d\right) .
$$

Thus, it remains to be seen whether the $1 / d$ term is also asymptotically sharp for large $d$.

\section{REFERENCES}

[C] J. Cheeger, A lower bound for the smallest eigenvalue of the Laplacian, in Problems in Analysis, A Symposium in honor of S. Bochner, Princeton University Press, 1970, 195-199.

[L] P. Li, A lower bound for the first eigenvalue of the Laplacian on a compact Riemannian manifold, Indiana Univ. Math. J., Vol. 28 (1979), 1013-1019.

[LY] P. Li and S.-T. Yau, Eigenvalues of a compact Riemannian manifold, AMS Proc. Symp. Pure Math. 36 (1980), 205-239.

[LZ] A. Lichnerowicz, Géometrie des groupes de transformations, Dunod, Paris, 1958.

[O] M. Obata, Certain Condition for a Riemannian manifold to be isometric with a sphere, J. Math. Soc. Japan 14 (1962), 333-340.

[SY] R. Schoen and S.-T. Yau, Lectures on Differential Geometry, International Press, 1994, Chapter 3.

[Y] D.-G. Yang, Lower Bound Estimates of the First Eigenvalue for Compact Manifolds with Positive Ricci Curvature, 1996 (preprint).

[ZY] J. Zhong and H. Yang, On the estimate of the first eigenvalue of a compact Riemannian manifold, Scientia, Sinica, vol XXVII, No. 12 (1984), 1265-1273. 
Department of Mathematics, Tulane University, New Orleans, LA 70118

E-mail address: kalka@math.tulane.edu

Department of Mathematics, Harvard University, Cambridge, MA 02138

E-mail address: mann@abel.math.harvard.edu

Department of Mathematics, Tulane University, New Orleans, LA 70118

E-mail address: dgy@math.tulane.edu

Department of Mathematics, Massachusetts Institute of Technology, Cambridge, MA 02139

E-mail address: azinger@mit.edu 